\title{
Is deception emotional? An emotion-driven predictive approach
}

\author{
Shahin Amiriparian ${ }^{1,2}$, Jouni Pohjalainen ${ }^{1}$, Erik Marchi ${ }^{1}$, \\ Sergey Pugachevskiy ${ }^{1}$, Björn Schuller ${ }^{1,3}$ \\ ${ }^{1}$ Chair of Complex and Intelligent Systems, University of Passau, Germany \\ ${ }^{2}$ Machine Intelligence \& Signal Processing Group, Technische Universität München, Germany \\ ${ }^{3}$ Machine Learning Group, Imperial College London, U.K. \\ shahin.amiriparianduni-passau.de
}

\begin{abstract}
In this paper, we propose a method for automatically detecting deceptive speech by relying on predicted scores derived from emotion dimensions such as arousal, valence, regulation, and emotion categories. The scores are derived from task-dependent models trained on the GEMEP emotional speech database. Inputs from the INTERSPEECH 2016 Computational Paralinguistics Deception sub-challenge are processed to obtain predictions of emotion attributes and associated scores that are then used as features in detecting deception. We show that using the new emotion-related features, it is possible to improve upon the challenge baseline.
\end{abstract}

Index Terms: computational paralinguistics, emotion, deception

\section{Introduction}

Deception is generally defined as "to cause to believe what is false" or "a deliberate attempt to mislead others" [1]. For centuries, practitioners and laypersons [2] have been interested in the question, do people behave in discernibly different ways when they are lying compared with when they are telling the truth? Assuming this to be the case leads to a practical challenge - detecting deception - which is a well-known task for its difficulty also for humans to perform reliably and consistently.

Detecting deception has long been important in the domains of psychology, law enforcement and other government agencies, international business, national security and research. Most scientific works and experimental studies focus on behavioural and visual cues to deception, such as facial expressions [3], on traditional biometric cues used in polygraphy $[4,5,6]$ or on body gestures $[7,8]$. An improvement in detecting deception can be made by analysing non-verbal cues like voice, verbal style or facial expressions: during talking, acting and especially while telling a lie, micro-expressions occur involuntary and express concealed emotions [9].

Recent studies have focussed on computational linguistics by developing intelligent systems in the scope of distinguishing between deceptive and non-deceptive speech using machine learning techniques $[10,11,12]$. However, as in every machine learning approach, the accuracy of these systems relies highly on the quality and quantity of the available data and annotation [13]. In addition to these factors, several studies investigated how emotions are influencing facial and vocal expression in a plethora of domains such as intelligent user interfaces [14], human-human interaction [15], human-robot interaction [16], human-computer interaction $[17,18]$, assistive in-car systems [19] and automatic speech emotion recognition [20, 21, 22].
Zuckerman et al. [4] proposed that truth tellers show less undifferentiated arousal than liars. Lying is often indicated by increased blinking, greater pupil dilation and/or higher tone of voice [4]. Hansen [23] describes characteristics of speech under stress and high arousal, which manifest themselves similarly in terms of acoustic parameters. This is caused by changes in speech production due to physiological effects [24, 25].

These studies corroborate our assumption that emotions and in particular arousal - are playing an important role in a deceptive expression. It has been observed that the performance of emotional lie detection (based on micro-expression training tools or subtle-expression training tools) is higher than that of unemotional lie detection [26]. It is also theoretically and experimentally more accurate and defensible to interpret arousal-related scores as indicative of deceptive speech $[27,28,29,30]$.

This paper describes our approach towards the Deception sub-challenge as part of the INTERSPEECH 2016 Computational Paralinguistics Challenge (ComParE) [31]. Our approach relies on predicted emotion-related attributes, such as arousal (activation), valence, regulation, and emotion categories. These high-level features are then used as new derived attribute vectors for detecting deception. If our assumption is correct that emotional cues are highly correlated to deception, then a system relying on emotion-derived features can be implemented to detect deception. Furthermore, it has to be noted that, labelled emotional speech databases are comparably more widely available than databases containing deceptive speech.

This paper is structured as follows. Firstly, the proposed system is introduced in Section 2. Then, Section 3 demonstrates the experimental set-up followed by extensive evaluations. Finally, conclusions are drawn and outlook is presented in Section 4 .

\section{Proposed System}

An overview of the proposed system is depicted in Fig. 1. It consists of two main functional components: 1) feature generation which involves classifiers for the emotional attributes arousal, valence, regulation and emotion classes, producing emotion-derived features, and 2) the main binary classifier which uses the emotion-related features and is trained to discriminate deceptive vs non-deceptive speech.

In the present work, feature generation is implemented by training a set of $k$-Nearest-Neighbour $(k \mathrm{NN})$ classifiers, each operating on a specific task, namely arousal, valence, regulation and emotion classes. Each of the four predictions is associated 
with two relevance values. Feature vectors are thus composed of 12 attributes: four predictions, each associated with two relevance values. They are used in the main classification stage both for the training and detection phase. For the main classification system, we apply both $k \mathrm{NN}$ and support vector machines (SVMs). In the following sections, these components are described in more detail.

\subsection{Emotion-driven features}

As depicted in Fig. 1, each of the four emotion tasks - arousal $(A)$, valence $(\mathrm{V})$, regulation $(\mathrm{R})$, and emotion $(\mathrm{E})$ - is predicted using a $k \mathrm{NN}$ classifier trained on the Geneva Multimodal Emotion Portrayals (GEMEP) emotional speech database [32]. These classifiers are built using the INTERSPEECH 2013 ComParE feature set [33], which is the same set of features as provided for the Deception sub-challenge. However, we also apply feature selection based on mutual information in order to find an optimised and reduced feature subset for each task. In addition to the predicted discrete-valued labels, each emotion attribute analyser outputs two continuous-valued scores: $z_{X}$, $X \in\{A, V, R, E\}$, the average distance of each data point to the predicted (majority) class among its $k$ nearest neighbours, and $w_{X}, X \in\{A, V, R, E\}$, the average distance to all the $k$ nearest neighbours (in the task-specific feature space).

The feature selection method chosen for each of the four emotional attributes is to rank the features based on their mutual information (MI) with the attribute label [34]. This is a simple approach which does not consider feature interdependencies and is thus not designed to obtain compact feature sets, unlike for example the popular, similarly mutual-information-based, minimum-redundancemaximal-relevance (MRMR) method [35]. Nevertheless, the MI feature scoring method, when combined with a reasonable way of deciding upon the number of features to select, has shown good performance in similar paralinguistic tasks, outperforming typical approaches such as MRMR and sequential forward selection [34]. The features are ranked in descending order of the mutual information

$$
M I=\sum_{y \in Y} \sum_{z \in Z} p(y, z) \log \left(\frac{p(y, z)}{p(y) p(z)}\right),
$$

where $Y$ is the set of discrete values of a quantised feature and $Z$ is the set of class labels. In previous work, the feature-specific quantisation scale to produce $Y$ has been adaptively determined in such a manner that each quantisation bin contains roughly the same number of samples over the data set under study [34]. In the MRMR method, a three-level quantisation scale with limits at one standard deviation on either side of the mean value is used [35]. In the present work, we have experimented with the aforementioned sample-count-equalising quantisation approach [34], which has a fixed number of quantisation levels, but have obtained better results with a one-dimensional clustering approach that automatically chooses the number of clusters/quantisation levels. It increases the number of clusters one by one and stops at the first point where the rate of decrease of total squared quantisation error, obtained while increasing the number of quantisation levels, has started to diminish noticeably. The goal here is to locate the first obvious turning point, after which adding more clusters does not improve the modelling of the data as much any more [36]. This is illustrated in Fig. 2.

The underlying motivation is that the method should explicitly favour such features, whose observed samples form distinct clusters that are in agreement with the labeling - assuming that such features do exist in the base feature set. This adds an unsupervised learning aspect to the feature selection approach. The maximum number of quantisation levels $|Y|$ considered in this process has been experimentally chosen as 10 based on the Development set performance of the deception detection system.

For each task, feature ranking by mutual information with respect to the labelling is performed on the Train (602 samples) and Development (216 samples) sets of GEMEP. The number of ranked features to use for classification is then determined by classifying the Test set (442 samples) using $k \mathrm{NN}$ via a grid search with $k$ ranging from 1 to 562 (the number of arousal and valence samples in the Train subset) and with the number of features $d \in\{50,100, \ldots, 5000\}$. The optimal number of features and optimal $k$ are chosen after median filtering the result grid of the Test set unweighted average recall (UAR) separately in both $k$ and $d$ dimensions and taking the minimum of the two. To obtain the final classifier for new data, we concatenate the Train, Development and Test sets of GEMEP and scale the optimal $k$ up in proportion to the increased training data size.

In $k \mathrm{NN}$ classification, the hypothesised class label for each test instance is determined as the label seen most frequently among the $k$ labelled training instances closest to the sample in terms of the Euclidean distance [37]. Despite its simplicity, $\mathrm{kNN}$ is a powerful pattern classification method that, given enough training data, can model complex nonlinear decision boundaries in the feature space [36]. It also lends itself well to generating nearest-neighbour-based relevance values for each class decision, as described earlier. However, $\mathrm{kNN}$ is susceptible to the effects of the curse of dimensionality $[36,37]$ and thus requires relatively high-quality features to give good results, which is also why we have focussed on improving feature selection for the emotion recognition tasks.

\subsection{Emotion classes}

In GEMEP, arousal and valence are labelled as positive, negative, and undefined, of which we do not use instances of the latter category. Regulation is labelled as high, low, and normal. The set of labels of emotion feature is \{amusement, anxiety, cold anger, despair, elation, hot anger, interest, other, panic fear, pleasure, pride, relief, sadness $\}$, from which we ignore the other class. These 12 labels, as shown in [32], have been mapped to arousal and valence categories.

\subsection{Deception detector}

As final stage classifier for the detection of deception, we apply both $k \mathrm{NN}$ and linear SVMs. Emotion-derived features are first normalised to zero mean and unit variance based on statistics of the training set. In deception detection, $k \mathrm{NN}$ is applied in the same form as in generating the emotion features. However, due to the aforementioned curse of dimensionality problem of $k \mathrm{NN}$, we limit the $k \mathrm{NN}$ classification experiments to low-dimensional feature sets consisting only of the newly generated features.

Adopting the Weka toolkit [38], SVMs with linear kernel are trained with the Sequential Minimal Optimization (SMO) algorithm. SVMs have been chosen as classifier since they are a well known standard method for emotion recognition due to their capability to handle high and low dimensional data. 


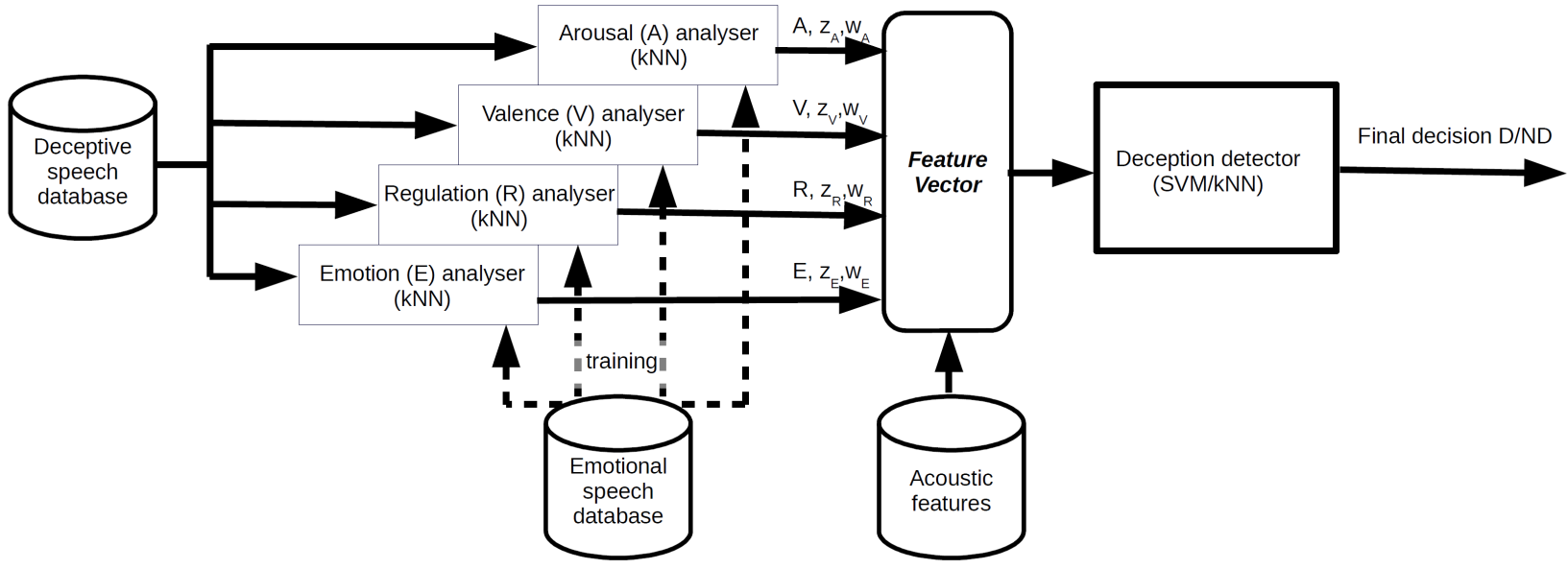

Figure 1: Flowchart of the deception recognising system: using acoustic and emotion-related features for detecting deceptive speech. The GEMEP emotional speech database is used for training the feature analysers.
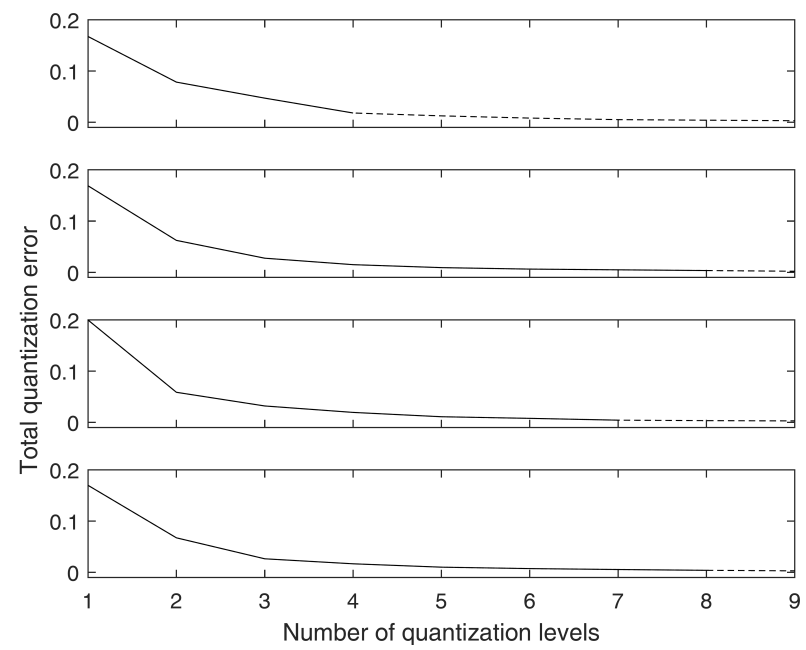

Figure 2: Behaviour of the total quantisation error as a function of quantisation levels for four features when constructing the feature-specific quantisation scale using $k$-means clustering. The end point of the solid line indicates the number of quantisation levels chosen by the present method, which looks for a point where the relative decrease rate of the quantisation error has stabilised.

\section{Experiments}

\subsection{Material and test setup}

Since all data sets are unbalanced (i.e., one class is underrepresented in the data), the unweighted average recall (UAR) of the classes is used as the scoring measure. The SVM training has been performed at different complexity values $C \in$ $\{0.004,0.005,30,80,0.2,9,3,90\}$.

According to the guidelines of the INTERSPEECH 2016 ComParE Deception Sub-Challenge, we apply the Deceptive Speech Database (DSD) created at the University of Arizona which has been divided for the challenge into a Train (182 deceptive, 390 non-deceptive instances), Development (129 deceptive, 357 non-deceptive) and Test (121 deceptive, 376 non- deceptive) set.

Firstly, we extract the 12 emotion-based features for all of the material, based on the 6373 features used by the baseline system. Then, our approach is to rely only on these extracted features. Firstly, we train our models with emotion features extracted from the Train set and classify the Development set. Using this setup, we tune the classifier parameters (complexity for linear-kernel SVM and number of neighbours for $k \mathrm{NN}$ ). Next, we retrain the most promising model configurations with the same emotion features from combined Train and Development set and aim to classify the Test data.

\subsection{Results}

Fig. 3 shows the behaviour of both classifiers for selected feature sets when the main parameter (the number of neighbours $k$ for $k \mathrm{NN}$, the complexity $C$ for SVM) is varied. Some observations can be made. Among arousal, valence and regulation, arousal appears to be the strongest individual feature for identifying deception. With several small subsets of emotional features (that include arousal), good deception detection performance, clearly over the Development set baseline, can be achieved in a stable manner over large intervals of the tuning parameter. We can also observe that the quality of the features is not classifier-dependent.

Table 1 shows the results on the Development set with both classifiers using various subsets of the 12 emotion-related features $\left(A, z_{A}, w_{A}, V, z_{V}, w_{V}, R, z_{R}, w_{R}, E, z_{E}, w_{E}\right)$ by themselves, and then the results of the SVM classifier using fusion of the 6373 baseline features with the emotion features. From the former approach, it is seen that the Development set baseline can be exceeded using the emotion features only. The latter approach leads to improved performance on the Test set, even though one might expect the large number of the baseline acoustic features to dominate the decision. This finding further suggests that the proposed emotion features have a high discriminative power in the deception classification task. Of the emotionrelated features, arousal by itself gives the best performance. However, combined with the baseline feature set extracted using openSMILE [39], arousal leads to less improvement over the baseline than valence. Compared to valence, the benefit of adding an arousal class of features appears to be reduced due to the large amount of acoustic features. 

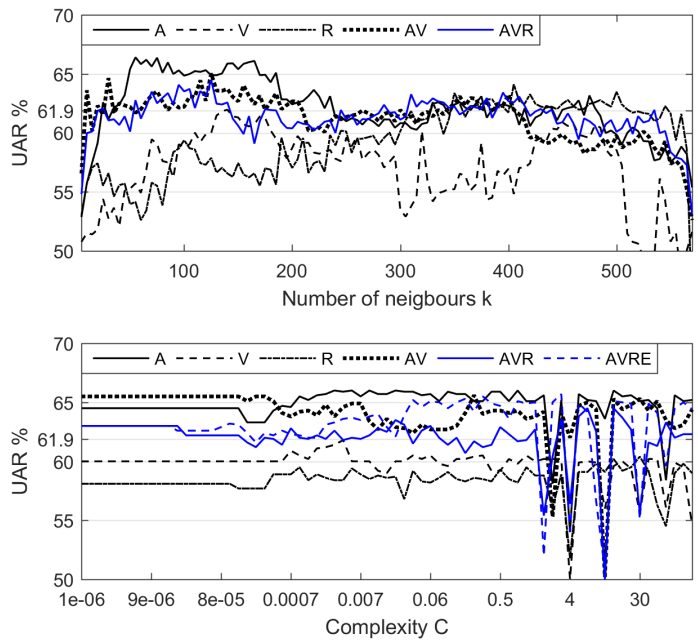

Figure 3: Unweighted average recall (UAR) for small subsets of emotional features arousal $(A)$, valence $(V)$, regulation $(R)$ and emotion category (E) using two classifiers, $k N N$ (upper panel) and SVM (lower panel). Each of the four feature classes involves a predicted categorical label and two continuous-valued scores reflecting the prediction confidence. The deception classifiers are evaluated by training on the Train subset and predicting the Development subset, using a wide range of the relevant tuning parameters (number of neighbours $k$ for $k N N$ and complexity $C$ for SVM). Results are shown for values of $k$ that are multiples of 5 and for the same values of $C$ as used in [31].

\section{Conclusions}

Application of emotion-related features in detecting deceptive speech was studied. We trained classifiers for categorical emotion attributes on an emotional speech database and applied those on the deceptive speech data in order to generate emotionrelated features. We then used these in the deception classification task in combination with different pattern classification methods.

We showed that, the emotional features have a relatively high predictive power in the deception task even when used by themselves. Remarkably, by means of fusion of the challenge baseline feature set (6373 features) with a small number of automatically generated descriptors related to, e.g., arousal, valence or emotion, we managed to exceed the Test set baseline of the challenge. These findings imply that emotional attributes, even ones generated by machine learning systems trained on separate data, have considerable potential for detecting deceptive speech. More generally, the results obtained support the feasibility of utilising labelled paralinguistic data in solving related problems, for which annotated data is scarcely available.

The approach chosen in this study for utilising emotion analysis resources - especially labelled emotion databases - for detecting deception was to try to produce high-quality features which contain information on deception, as well. Our results show that this approach holds potential for future development of deception detection systems.

Future studies can also investigate whether other speaker states are also encoding relevant information for detecting deceptive speech. For example, likeability and interest might be interesting dimensions to look at for this purpose.
Table 1: Deception classification performance on the Development set (\% UAR). The emotion feature combinations are denoted by the shorthand notation where, for example, A' indicates the pair of features $\left(A, z_{A}\right)$ and $A$ " indicates the feature triplet $\left(A, z_{A}, w_{A}\right)$. ' 6373 ' indicates the complete baseline openSMILE (OS) feature set of 6373 features.

\begin{tabular}{|c|c|c|c|c|}
\hline \multicolumn{2}{|c|}{$\begin{array}{c}\text { Classifier } \\
\text { and features }\end{array}$} & $\begin{array}{l}\text { Chosen } \\
\text { hyperparameter }\end{array}$ & $\begin{array}{l}\text { Development } \\
\text { maximum }\end{array}$ & $\begin{array}{l}\text { Test } \\
\text { trials }\end{array}$ \\
\hline \multirow[t]{7}{*}{$k \mathrm{NN}$} & $\mathrm{A}^{\prime}$ & $\mathrm{k}=185$ & 66.9 & N/A \\
\hline & V' & $\mathrm{k}=138$ & 61.8 & N/A \\
\hline & A" & $\mathrm{k}=57$ & 66.6 & N/A \\
\hline & V" & $\mathrm{k}=148$ & 62.0 & N/A \\
\hline & $A^{\prime} R^{\prime}$ & $\mathrm{k}=273$ & 65.6 & N/A \\
\hline & A"V", & $\mathrm{k}=119$ & 65.1 & N/A \\
\hline & A"V"R" & $\mathrm{k}=97$ & 64.8 & N/A \\
\hline \multirow[t]{10}{*}{ SVM } & A" & $\mathrm{C}=0.004$ & 66.0 & N/A \\
\hline & V" & $\mathrm{C}=0.005$ & 61.8 & N/A \\
\hline & R” & $\mathrm{C}=30$ & 60.4 & N/A \\
\hline & V"E" & $\mathrm{C}=9$ & 61.3 & N/A \\
\hline & A"V" & $\mathrm{C}=0.2$ & 65.6 & N/A \\
\hline & A'E" & $\mathrm{C}=3$ & 66.5 & N/A \\
\hline & A"V’E" & $\mathrm{C}=0.9$ & 65.7 & N/A \\
\hline & A"V"R" & $\mathrm{C}=9$ & 64.5 & N/A \\
\hline & A"V"R"E" & $\mathrm{C}=3$ & 65.7 & N/A \\
\hline & A'R'E”' & $\mathrm{C}=90$ & 67.7 & N/A \\
\hline \multicolumn{2}{|c|}{ Emotion + OS } & & & \\
\hline \multirow[t]{3}{*}{ SVM } & $A^{\prime \prime}+6373$ & $\mathrm{C}=10^{-4}$ & 65.2 & 68.8 \\
\hline & V" +6373 & $\mathrm{C}=10^{-4}$ & 66.1 & 68.9 \\
\hline & $E "+6373$ & $\mathrm{C}=10^{-4}$ & 62.1 & 68.9 \\
\hline \multicolumn{2}{|c|}{ Baseline } & & & \\
\hline SVM & 6373 & $\mathrm{C}=10^{-4}$ & 61.9 & 68.3 \\
\hline
\end{tabular}

\section{Acknowledgements}

The research leading to these results has received funding from the ECs Seventh Framework Programme through the ERC Starting Grant No. 338164 (iHEARu), and No. 688835 (RIA DE-ENIGMA).

\section{References}

[1] B. M. DePaulo, J. J. Lindsay, B. E. Malone, L. Muhlenbruck, K. Charlton, and H. Cooper, "Cues to deception." Psychological bulletin, vol. 129, no. 1, p. 74, 2003.

[2] P. V. Trovillo, “A history of lie detection," Journal of Criminal Law and Criminology (1931-1951), vol. 29, no. 6, pp. 848-881, 1939.

[3] P. Ekman and W. V. Friesen, "Detecting deception from the body or face." Journal of Personality and Social Psychology, vol. 29, no. 3, p. 288, 1974.

[4] M. Zuckerman, B. M. DePaulo, and R. Rosenthal, "Verbal and nonverbal communication of deception," Advances in experimental social psychology, vol. 14, no. 1, p. 59, 1981.

[5] F. S. Horvath, "Verbal and nonverbal clues to truth and deception during polygraph examinations." Journal of Police Science \& Administration, 1973.

[6] P. A. Granhag, A. Vrij, and B. Verschuere, Detecting Deception: Current Challenges and Cognitive Approaches. John Wiley \& Sons, 2015.

[7] J. K. Burgoon, D. B. Buller, A. S. Ebesu, and P. Rockwell, "Interpersonal deception: V. Accuracy in deception detection," Communications Monographs, vol. 61, no. 4, pp. 303-325, 1994. 
[8] A. Kleinsmith and N. Bianchi-Berthouze, "Affective body expression perception and recognition: A survey," Affective Computing, IEEE Transactions on, vol. 4, no. 1, pp. 15-33, 2013.

[9] J. P. Gaspar and M. E. Schweitzer, "The emotion deception model: a review of deception in negotiation and the role of emotion in deception," Negotiation and Conflict Management Research, vol. 6, no. 3, pp. 160-179, 2013.

[10] J. Hirschberg, S. Benus, J. M. Brenier, F. Enos, S. Friedman, S. Gilman, C. Girand, M. Graciarena, A. Kathol, L. Michaelis et al., "Distinguishing deceptive from non-deceptive speech." in INTERSPEECH, Lisbon, Portugal, 2005, pp. 1833-1836.

[11] S. Benus, F. Enos, J. Hirschberg, and E. Shriberg, "Pauses in deceptive speech," in Speech Prosody, vol. 18. Dresden, Germany: Proceedings in ISCA 3rd International Conference on Speech Prosody, 2006, pp. 2-5.

[12] M. Graciarena, E. Shriberg, A. Stolcke, F. Enos, J. Hirschberg, and S. Kajarekar, "Combining prosodic lexical and cepstral systems for deceptive speech detection," in Acoustics, Speech and Signal Processing, 2006. ICASSP 2006 Proceedings. 2006 IEEE International Conference on, vol. 1. Toulouse, France: IEEE, 2006

[13] S. Hantke, E. Marchi, and B. Schuller, "Introducing the Weighted Trustability Evaluator for Crowdsourcing Exemplified by Speaker Likability Classification," in Proceedings 10th Language Resources and Evaluation Conference (LREC 2016), ELRA. Portoroz, Slovenia: ELRA, May 2016.

[14] N. Sabouret, L. Paletta, B. Schuller, E. Marchi, H. Jones, and A. B. Youssef, "Intelligent User Interfaces in Digital Games for Empowerment and Inclusion," in Proceedings of the 12th International Conference on Advancement in Computer Entertainment Technology, ACE 2015, ACM. Iskandar, Malaysia: ACM, November 2015.

[15] V. Petrushin, "Emotion in speech: Recognition and application to call centers," in Proc. of Artificial Neural Networks in Engineering, vol. 710, St. Louis, MO, 1999, pp. 7-10.

[16] E. Marchi, F. Ringeval, and B. Schuller, "Voice-enabled Assistive Robots for Handling Autism Spectrum Conditions: An Examination of the Role of Prosody," in Speech and Automata in the Health Care, A. Neustein, Ed. Walter de Gruyter GmbH \& Co KG, 2014, pp. 207-236.

[17] B. Schuller, E. Marchi, S. Baron-Cohen, A. Lassalle, H. O’Reilly et al., "Recent Developments and Results of ASC-Inclusion: An Integrated Internet-Based Environment for Social Inclusion of Children with Autism Spectrum Conditions," in Proc. of IDGEI, Atlanta, GA, 2015.

[18] E. Marchi, B. Schuller, S. Baron-Cohen, A. Lassalle et al., "Voice Emotion Games: Language and Emotion in the Voice of Children with Autism Spectrum Condition," in Proc. of IDGEI, ACM. Atlanta, GA: ACM, March 2015.

[19] I. Abdić, L. Fridman, D. McDuff, E. Marchi, B. Reimer, and B. Schuller, "Driver Frustration Detection From Audio and Video," in Proceedings of the 25th International Joint Conference on Artificial Intelligence, IJCAI 2016. New York City, NY: IJCAI/AAAI, July 2016.

[20] F. Eyben, K. R. Scherer, B. W. Schuller, J. Sundberg, E. André, C. Busso, L. Y. Devillers, J. Epps, P. Laukka, S. S. Narayanan et al., "The Geneva minimalistic acoustic parameter set (GeMAPS) for voice research and affective computing," IEEE Transactions on Affective Computing, vol. 7, no. 2, pp. 190-202, 2016

[21] F. Ringeval, S. Amiriparian, F. Eyben, K. Scherer, and B. Schuller, "Emotion recognition in the wild: Incorporating voice and lip activity in multimodal decision-level fusion," in Proceedings of the 16th International Conference on Multimodal Interaction. Istanbul, Turkey: ACM, 2014, pp. 473-480.

[22] E. Marchi, B. Schuller, S. Baron-Cohen, O. Golan, S. Bölte, P. Arora, and R. Häb-Umbach, "Typicality and Emotion in the Voice of Children with Autism Spectrum Condition: Evidence
Across Three Languages," in Proceedings INTERSPEECH 2015, 16th Annual Conference of the International Speech Communication Association, ISCA. Dresden, Germany: ISCA, September 2015, pp. 115-119.

[23] J. H. L. Hansen, "Analysis and compensation of speech under stress and noise for environmental robustness in speech recognition," Speech communication, vol. 20, no. 1, pp. 151-173, 1996.

[24] J. Wagner, J. Kim, and E. André, "From physiological signals to emotions: Implementing and comparing selected methods for feature extraction and classification," in 2005 IEEE International Conference on Multimedia and Expo. Amsterdam, The Netherlands: IEEE, 2005, pp. 940-943.

[25] G. Zhou, J. H. L. Hansen, and J. F. Kaiser, "Nonlinear feature based classification of speech under stress," IEEE Transactions on Speech and Audio Processing, vol. 9, no. 3, pp. 201-216, 2001.

[26] G. Warren, E. Schertler, and P. Bull, "Detecting deception from emotional and unemotional cues," Journal of Nonverbal Behavior, vol. 33, no. 1, pp. 59-69, 2009.

[27] G. G. Sparks and J. O. Greene, "On the validity of nonverbal indicators as measures of physiological arousal: A response to Burgoon, Kelley, Newton, and Keeley-Dyreson." Human Communication Research, vol. 18, no. 3, pp. 445-471, 1992.

[28] R. Neiss, "Reconceptualizing arousal: psychobiological states in motor performance." Psychological Bulletin, vol. 103, no. 3, p. 345, 1988.

[29] P. Ekman, R. W. Levenson, and W. V. Friesen, "Autonomic nervous system activity distinguishes among emotions," Science, vol. 221 , no. 4616, pp. 1208-1210, 1983.

[30] M. A. DeTurck and G. R. Miller, "Deception and arousal: Isolating the behavioral correlates of deception." Human Communication Research, 1985.

[31] B. Schuller, S. Steidl, A. Batliner, J. Hirschberg, J. K. Burgoon, A. Baird, A. Elkins, Y. Zhang, E. Coutinho, and K. Evanini, "The INTERSPEECH 2016 Computational Paralinguistics Challenge: Deception, Sincerity \& Native Language," in Proceedings of INTERSPEECH, San Francisco, CA, 2016.

[32] T. Bänziger and K. R. Scherer, "Using actor portrayals to systematically study multimodal emotion expression: The GEMEP corpus," in Affective computing and intelligent interaction. Springer, 2007, pp. 476-487.

[33] B. Schuller, S. Steidl, A. Batliner, A. Vinciarelli, K. Scherer, F. Ringeval, M. Chetouani, F. Weninger, F. Eyben, E. Marchi, M. Mortillaro, H. Salamin, A. Polychroniou, F. Valente, and S. Kim, "The INTERSPEECH 2013 Computational Paralinguistics Challenge: Social Signals, Conflict, Emotion, Autism," in Proceedings of INTERSPEECH. Lyon, France: ISCA, 2013, pp. 148-152.

[34] J. Pohjalainen, O. Räsänen, and S. Kadioglu, "Feature selection methods and their combinations in high-dimensional classification of speaker likability, intelligibility and personality traits," Computer Speech and Language, vol. 29, no. 1, pp. 145-171, January 2015.

[35] H. Peng, F. Long, and C. Ding, "Feature selection based on mutual information: Criteria of max-dependency, max-relevance, and min-redundancy," IEEE Transactions on Pattern Analysis and Machine Intelligence, vol. 27, no. 8, pp. 1226-1238, August 2005.

[36] S. Theodoridis and K. Koutroumbas, Pattern Recognition, 2nd ed. Academic Press, 2003

[37] R. O. Duda, P. E. Hart, and D. G. Stork, Pattern Classification, 2nd ed. John Wiley and Sons Inc., 2001.

[38] M. Hall, E. Frank, G. Holmes, B. Pfahringer, P. Reutemann, and I. H. Witten, "The WEKA data mining software: an update," SIGKDD Explor. Newsl., vol. 11, no. 1, pp. 10-18, Nov. 2009.

[39] F. Eyben, F. Weninger, F. Gross, and B. Schuller, "Recent developments in openSMILE, the Munich open-source multimedia feature extractor," in Proceedings of the 21st ACM international conference on Multimedia. Barcelona, Spain: ACM, 2013, pp. 835-838. 